\title{
DW VECSEL - structure design and MBE fabrication
}

\author{
Agata Jasik, ${ }^{1 *}$ Jan Muszalski, ${ }^{1}$ Anna Wójcik-Jedlińska, ${ }^{1}$ Justyna Kubacka-Traczyk, ${ }^{1}$ Michał Wasiak, ${ }^{2}$ \\ Adam K. Sokót ${ }^{2}$ and Iwona Sankowska ${ }^{1}$ \\ ${ }^{1}$ Institute of Electron Technology, Al. Lotnikow 32/46, 00-668 Warszawa, Poland \\ ${ }^{2}$ Institute of Physics, Lodz University of Technology, ul. Wólczańska 219, 90-924 Łódź, Poland
}

Received August 08, 2013; accepted September 24, 2013; published September 30, 2013

\begin{abstract}
We demonstrate dual-wavelength emission from an optically pumped vertical-external-cavity surface-emitting laser. The active region containing two sets of quantum wells is divided into two sections with thicknesses providing comparable pump absorption. The sections are separated by an AlAs blocking layer to prevent carrier flow between them. The structure was grown by molecular beam epitaxy. The continuous wave operation was simultaneously detected at $957 \mathrm{~nm}$ and $1011 \mathrm{~nm}$.
\end{abstract}

Dual-wavelength vertical external-cavity surface emitting lasers (DW VECSELs) were theoretically proposed by Morozov et al. in 2004 [1]. Shortly after that a simultaneous dual-wavelength emission from an optically pumped VECSEL was demonstrated for the first time [2] In that device the gain region was divided into three sections to provide uniform filling of quantum wells by carriers: a long-wavelength section placed between two short-wavelength sections [2-3]. To prevent interaction between optical fields, the deep wells emitting a long wavelength were located near the electric field nodes of short-wavelength radiation [2-4]. This has been also achieved by introducing a filter between the regions of wells with different depths [5-6]. Both designs are very complex and difficult to fabricate. The desired laser structure should contain a two-sectional gain region and be relatively thin.

In this paper we report simple geometry of an opticallypumped dual-wavelength VECSEL in comparison to reported designs, providing comparable threshold power for both wavelengths [1-7].

A typical VECSEL heterostructure consists of a distributed Bragg reflector (DBR) and an active region of quantum wells (QWs) or quantum dots (QDs) enclosed in a microcavity. The design of a DW VECSEL heterostructure is more demanding since the range of DBR high reflectivity has to be broad enough to enclose a laser resonator at two wavelengths, and the microcavity has to hold two different active regions. The active regions must be independent, i.e. two sets of QWs are needed and each of them has to interact only with its own wave. Those two sets of QWs have to provide similar gain

\footnotetext{
*E-mail: ajasik@ite.waw.pl
}

in order to switch the laser emission on at both wavelengths at the same pump power. The carrier filling of both sets of QWs should be similar. For a low threshold emission the cavity should be resonant for both wavelengths.

The dual-wavelength VECSEL heterostructure presented here consists of the AlAs/GaAs DBR and two active regions of $8-\mathrm{nm} \mathrm{In}_{\mathrm{X}} \mathrm{Ga}_{1-\mathrm{X}} \mathrm{As} / \mathrm{GaAs}$ quantum wells emitting at $956 \mathrm{~nm}(\mathrm{x}=18 \%)$ and $1011 \mathrm{~nm}(\mathrm{x}=24 \%)$. The structure is finished by a window layer of $\mathrm{Al}_{0.5} \mathrm{Ga}_{0.5} \mathrm{As}$ capped by a GaAs layer of $10 \mathrm{~nm}$. Figure 1 presents the refractive index and optical field intensities for our DW VECSEL.

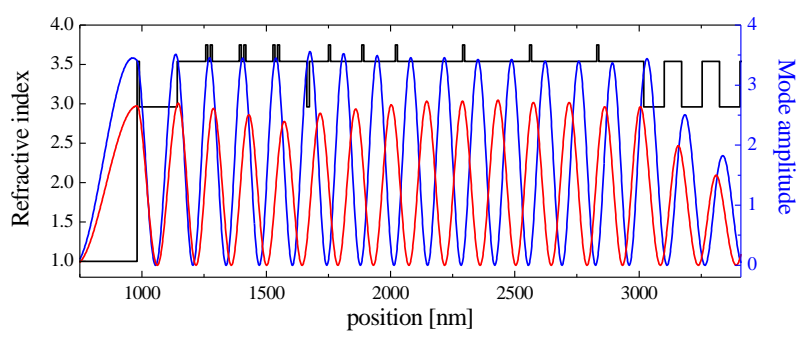

Fig. 1. The refractive index (black color) and the mode amplitude for $\lambda_{S}=957 \mathrm{~nm}$ (blue color) and $\lambda_{\mathrm{L}}=1011 \mathrm{~nm}$ (red color) for dual-wavelength

VECSEL. Short-wavelength radiation $\lambda_{S}$ is emitted from shallow quantum wells $\mathrm{QW}^{\mathrm{S}}$, the long-wavelength radiation $\lambda_{\mathrm{L}}$ is emitted from deep quantum wells $\mathrm{QW}^{\mathrm{L}}$.

The DBR was designed with a high reflectivity plateau central wavelength of $\lambda=980 \mathrm{~nm}$ and it consists of 27 pairs of $\lambda / 4$-thick GaAs and AlAs layers. For this mirror, the spectral range of high reflectivity exceeds $100 \mathrm{~nm}$. This allows to obtain large spectral separation of emitted wavelengths $(55 \mathrm{~nm})$. A $13.5 \times \lambda$ GaAs microcavity was designed on the top of DBR and shared by two active regions. The two sets of QWs are placed in subsequent antinodes of the proper waves. However, the quantum wells $\left(\mathrm{QW}^{\mathrm{L}}\right)$ which provide gain at the longer wavelength $\left(\lambda_{\mathrm{L}}\right)$ absorb the light emitted by the quantum wells $\left(\mathrm{QW}^{\mathrm{S}}\right)$ designed for emission at a shorter wavelength $\left(\lambda_{S}\right)$. Such absorption has to be strictly avoided. Because of that, for proper laser operation the $\mathrm{QW}^{\mathrm{L}}$ were shifted slightly out of antinode position of the $\lambda_{\mathrm{L}}$ to the nodes of the $\lambda_{\mathrm{S}}$. This 
was at the expense of the value of a longitudinal confinement factor of $\mathrm{QW}^{\mathrm{L}}$ [8]. In Fig. 2, the longitudinal confinement factor for both sets of quantum wells is shown. The longitudinal confinement factor for $\mathrm{QW}^{\mathrm{L}}$ vanishes at the emission wavelength of the $\mathrm{QW}^{\mathrm{S}}$. By contrast, the high confinement factor of $\mathrm{QW}^{\mathrm{S}}$ at the emission wavelength of $\lambda_{\mathrm{L}}$ is not an obstacle since the $\mathrm{QW}^{\mathrm{S}}$ do not interact with the light emitted at $\lambda_{\mathrm{L}}$ due to larger energy separation of quantum levels in these quantum wells.

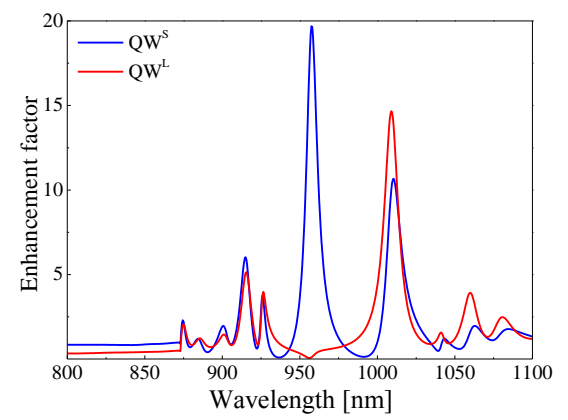

Fig. 2. The longitudinal confinement factor for two sets of quantum wells emitting at $\lambda_{S}=956 \mathrm{~nm}$ and $\lambda_{\mathrm{L}}=1011 \mathrm{~nm}$. The QW $\mathrm{QW}^{\mathrm{L}}$ vanishes at the emission wavelength of $\mathrm{QW}^{\mathrm{S}}$.

The two sets of QWs have to be separated, i.e. the cavity has to be divided into two parts by a barrier. A barrier is needed in order to prevent the diffusion of carriers between two sets of $\mathrm{QWs}$, otherwise the $\mathrm{QW}$ designed for the emission at the longer wavelength would be privileged. The lower energy of confined levels results in a larger barrier height, thus a slower escape of thermally activated carriers. The position of the barrier was adjusted to assure an equal number of photo-carriers in each part assuming an exponential decrease of the pump power. Consequently, the part of the microcavity on the side of the wafer surface is much shorter than on the DBR side. Since there are only three antinodes in the short part of the cavity, in which three double $\mathrm{QW}^{\mathrm{S}}$ were positioned. The second part is much longer so six single $\mathrm{QW}^{\mathrm{L}}$ were positioned in a sequence of 111010101 , where "1" stands for a single $\mathrm{QW}^{\mathrm{L}}$ in the antinode and "0" for a missing position (Fig. 1).

The structure of DW VECSEL was grown by molecular beam epitaxy. The Riber $32 \mathrm{P}$ reactor was equipped with standard effusion cells for group III elements ( $\mathrm{Ga}, \mathrm{Al}$ and In) and valved cracking cell for arsenic. The growth temperature was $530^{\circ} \mathrm{C}$ and kept constant during the whole epitaxial process. To keep fabrication accuracy within $0.5 \%$, the growth rate was controlled in two ways: in situ by reflection high energy electron diffraction RHEED oscillations and ex situ by high resolution X-ray diffractometry HR XRD. The optimization of epitaxial growth of the DW VECSEL structure was divided into three stages of: (1) the active region consisting of two types of QWs and (2) the DBR structures and (3) the VECSEL structures emitting one wavelength. As a result, the DW VECSEL has been grown. The as-grown structure was characterized by photoluminescence and reflectance spectroscopy. The results are presented in Fig. 3.

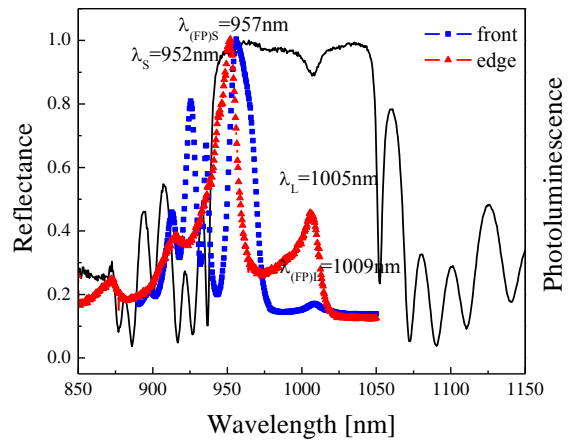

Fig. 3. The reflectance R and PL spectra for DW VECSEL. The symbols are for PL spectra taken from the surface (squares) and from the edge (triangles). $\lambda_{S}=952 \mathrm{~nm}$ and $\lambda_{\mathrm{L}}=1005 \mathrm{~nm}$ - emission wavelengths originate from shallow and deep QWs, respectively. The F-P resonances are at wavelengths of $\lambda_{(\mathrm{F}-\mathrm{P}) \mathrm{S}}=957 \mathrm{~nm}$ and $\lambda_{(\mathrm{F}-\mathrm{P}) \mathrm{L}}=1009 \mathrm{~nm}$.

The PL spectrum measured from the surface and the dips on reflectance indicate wavelengths of F-P resonances, which are at $957 \mathrm{~nm}$ and $1009 \mathrm{~nm}$, whereas the PL spectrum taken from the edge of a structure shows emission from the shallow and deep quantum wells at wavelengths of $\lambda_{S}=952 \mathrm{~nm}$ and $\lambda_{\mathrm{L}}=1005 \mathrm{~nm}$, respectively. It means that the structure was made with an accuracy higher than $0.5 \%$.

Finally, the lasing properties of as-grown samples were tested using a simple linear resonator. An external laser cavity was formed between the DBR in DW VECSEL structure and the external output coupler (OC). The OC curvature and the transparency were $9.0 \mathrm{~cm}$ and $2 \%$, respectively. A laser diode emitting at $808 \mathrm{~nm}$ was used for optical pumping. The laser beam was directed at the DW VECSEL chip at an angle of $30^{\circ}$ from the optical axis of the laser cavity and was focused on the chip surface to a spot size of $60 \mu \mathrm{m}$. The unprocessed DW VECSEL chip glued by heat conductive silicon paste on a heat sink was supported and thermally stabilized by the Peltier element. A water-cooling system was used to further enhance heat dissipation. A PM100 laser power meter equipped with an S130C photodiode power sensor was used to measure the output power. The setup of the DW VECSEL cavity is shown in Fig. 4. 


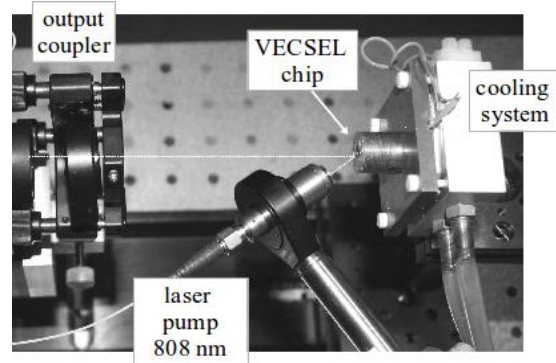

Fig. 4. DW VECSEL cavity setup.

Figure 5(a) presents lasing spectra measured at a pump power of $700 \mathrm{~mW}$. Both the wavelengths lased in continuous-wave mode. The wavelength spectral separation was $55 \mathrm{~nm}$. Such large spectral separation is not achievable by other intracavity techniques [9].
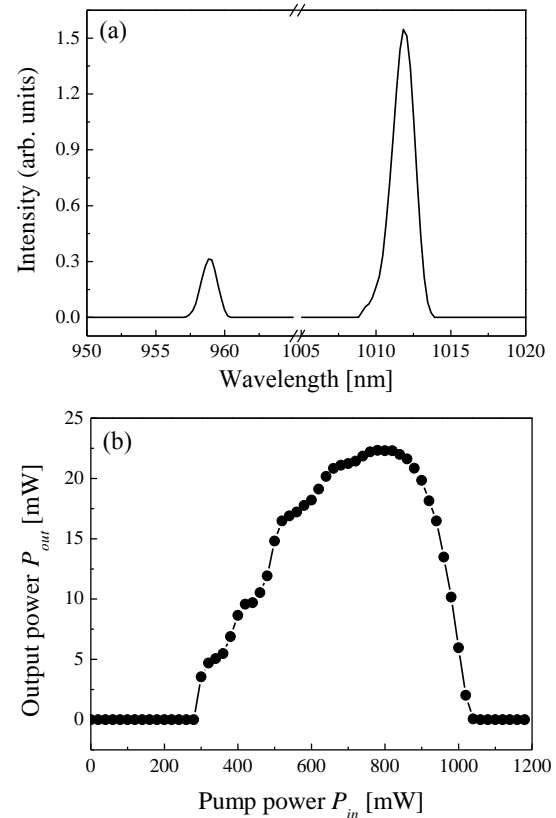

Fig. 5. The spectral (a) and the output power vs. the pump power (b) characteristics for DW VECSEL. The lasing spectra were measured at pump power of $700 \mathrm{~mW}$. The output coupler: $\mathrm{R}=90 \mathrm{~mm}, \mathrm{~T}=2 \%$. The temperature on the heat sink was $-5^{\circ} \mathrm{C}$.

The output power $P_{\text {out }}$ versus power of pump $P_{\text {in }}$ for unseparated wavelengths is shown in Fig. 5(b). The power ratio for longer and shorter wavelengths is comparable to the intensity ratio [Fig. 5(a)] and equals 5.0. The optical efficiency from the power characteristic, taking into account the reflection from the DW VECSEL surface, is about $5 \%$. Notice that this result is for the as-grown structure with poor heat dissipation. The optical efficiency of $11 \%$ can be estimated from the characteristic presented in Ref. [10] but for the processed DW VECSEL.

We have designed and fabricated a monolithic dualwavelength vertical-cavity surface-emitting laser which is capable to provide gain simultaneously at two separated wavelengths. We observed continuous-wave operation for both frequencies. The advantage of our design is its simplicity - two-sectional cavity (instead of three, [5]) without any pass-filters, which significantly increases the total thickness of a laser and hence the complexity and difficulty of fabrication.

We believe DW VECSELs are suitable devices for intracavity difference frequency generation.

This work was partially supported by the National Science Centre (NCN) under project 3606/B/T02/2009/36, the National Centre for Research and Development (NCBiR) under projects 02-0009-10/2011 and 02-0023-06.

\section{References}

[1] Y. Morozov, I. Nefedov, V. Aleshkin, Semiconductors 38, 1350 (2004).

[2] T. Leinonen, Y.A. Morozov, A. Harkonen, M. Pessa, IEEE Photon. Technol. Lett. 17, 2508 (2005)

[3] Y.A. Morozov, T. Leinonen, A. Härkönen, M. Pessa, IEEE J. Quantum Electron. 42, 1055 (2006)

[4] Y.A. Morozov, I.S. Nefedov, T. Leinonen, M.Y. Morozov, Semiconductors 42, 463 (2008)

[5] T. Leinonen, S. Ranta, A. Laakso, Y.A. Morozov, M. Saarinen, M. Pessa, Opt. Expr. 15, 13451 (2007).

[6] M.Y. Morozov, Y.A. Morozov, V.V. Popov, Semiconductors 43, 382 (2009).

[7] Y.A. Morozov, M.Y. Morozov, I.V. Krasnikova, Tech. Phys. Lett. 37, 1112 (2011).

[8] A.C. Troppera, S. Hoogland, Progr. Quant. Electron. 30, 1 (2006).

[9] L. Fan, M. Fallahi, J. Hader, A.R. Zakharian, J.V. Moloney, W. Stolz, S.W. Koch, R. Bedford, J.T. Murray, Appl. Phys.Lett. 90, 181124 (2007).

[10] S. Ranta, T. Leinonen, A. Härkönen, A. Laakso, Y. Morozov, M. Pessa, Lithuanian J. Phys. 50, 27 (2010). 